\title{
Minimally invasive, beating heart tricuspid valve surgery in a redo case
}

\author{
Martin Misfeld ${ }^{1}$, Piroze Davierwala ${ }^{1}$, Jörgen Banusch ${ }^{2}$, Jörg Ender ${ }^{2}$, Friedrich-Wilhelm Mohr ${ }^{1}$, Bettina \\ Pfannmüller ${ }^{1}$ \\ ${ }^{1}$ Department of Cardiac Surgery, ${ }^{2}$ Department of Anesthesiology, Heart Center, University of Leipzig, Germany \\ Correspondence to: Martin Misfeld, MD, PhD. Department of Cardiac Surgery, Heart Center, University of Leipzig Struempellstrasse 39 , 04289 \\ Leipzig, Germany. Email: martinmisfeld@yahoo.com.
}

Submitted Apr 10, 2017. Accepted for publication Apr 20, 2017.

doi: 10.21037/acs.2017.05.10

View this article at: http://dx.doi.org/10.21037/acs.2017.05.10

\section{Clinical vignette}

A 73-year-old female patient was admitted to our hospital with tricuspid valve (TV) regurgitation grade IV. Past medical history revealed previous mechanical mitral valve replacement and implantation of a pacemaker. Clinically she had typical signs of chronic congestive right heart failure. Massive edema of the lower legs were detected during clinical examination. Preoperative echo confirmed severe TV regurgitation and normal function of the mechanical mitral valve. Coronary artery disease was excluded by coronary angiogram. CT scan demonstrated massive dilatation of the right heart chambers. The right ventricle was in part stuck to the sternum (Figure 1). CT scan further showed "cirrhose cardiaque" due to chronic right heart congestion. She was referred for reoperation with TV repair or replacement.

\section{Preoperative planning}

Median sternotomy for redo surgery is the standard access, and also in cases where the TV has to be addressed. However, as minimally invasive surgery for the atrioventricular valves and/or atrial septal defects as well as ablation therapy for atrial fibrillation has been the standard surgical access in our department for years (1-4), this technique has also been used in reoperations (5).

For TV surgery, avoidance of median sternotomy offers specific advantages. It avoids the risk of right heart damage during median sternotomy. In the case presented, this risk was considerable high due to the close adherence of the right ventricle to the sternum. Minimizing dissection of the heart also reduces the area of potential bleeding sites. Preserving the integrity of the massive dilated right ventricle in the surrounding tissue may also prevents further dilatation after TV repair or replacement and, therefore, right heart failure (5). Additionally, the risk of bleeding is increased due to chronic liver congestion with impaired liver function and synthesis of coagulation factors.

We also prefer reoperations for TV surgery on the beating heart when possible as our previous results have shown favorable outcome with this technique (6)

\section{Surgical technique}

\section{Cannulation}

Following induction of anesthesia, a $16 \mathrm{~F}$ or $18 \mathrm{~F}$ arterial cannula (Edwards Lifesciences, Irvine, CA, USA) is inserted into the right internal jugular vein by the anesthetist (Figure 2). The size depends on the size of the internal jugular vein, as judged by ultrasound scanning. Location and insertion of the cannula is performed under direct and transesophageal echo (TOE) guidance and control. A bubble test confirms adequate position of the cannula. An additional 14 F Fogarty catheter (Edwards Lifescience, Irvine, CA, USA) is inserted through the superior vena cava (SVC) and initially positioned in the right atrium (RA) for later blockage of the SVC and, therefore induction of total cardiopulmonary bypass (CPB). The patient is then transferred from the induction room to the operation room.

The right femoral artery and vein are our standard target vessels in all minimally invasive procedures through a right antero-lateral incision (Figure 3). Following an oblique 


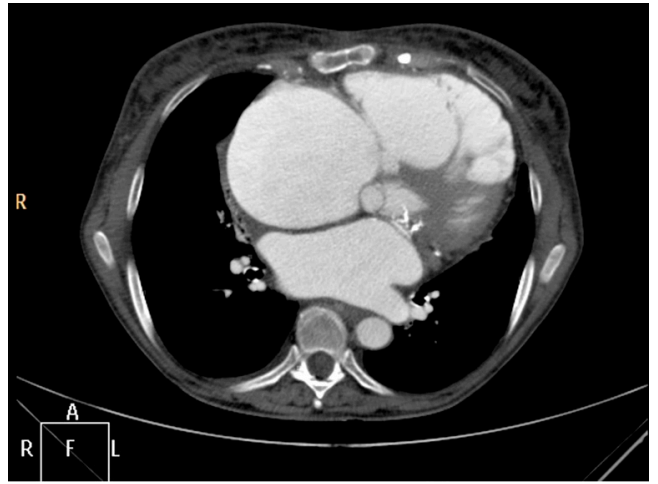

Figure 1 Preoperative computer tomography scan revealed right heard enlargement and adhesion of the right ventricle to the retrosternal space.

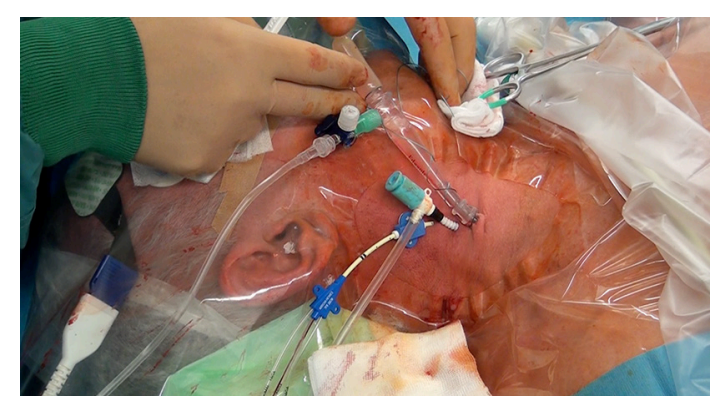

Figure 2 Venous cannulation of the right internal jugular vein.

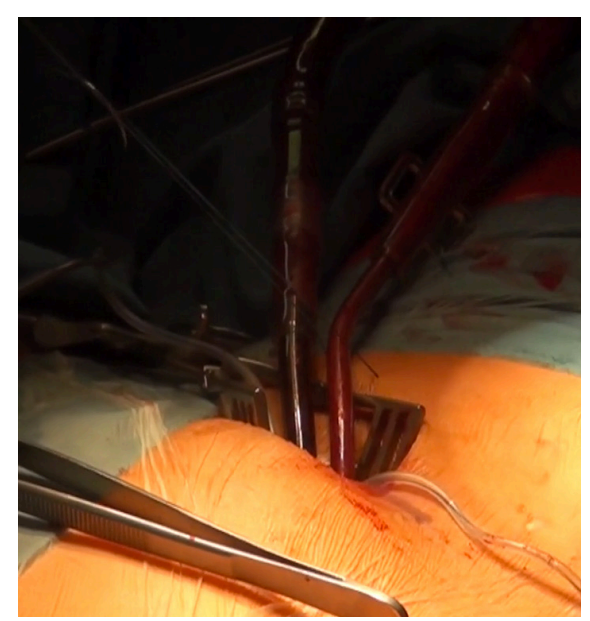

Figure 3 Cannulation of the femoral vein and artery.

limited skin incision, the right femoral artery and vein are dissected superficially. The quality of the artery is judged by palpation. A 4/0 monofilament pure-string suture is placed on each vessel. We start with venous cannulation as this vessel might be positioned slightly posterior to the artery. Using the Seldinger technique, the guiding wire is advanced into the SVC under echo guidance. Dilatators of increasing size are used, before a 22 to $25 \mathrm{~F}$ venous cannula (Edwards Lifescience, Irvine, CA, USA) is advanced into the SVC, also under close echo control. Care has to be taken not to advance the cannula against any resistance. Arterial cannulation of a 16 to $22 \mathrm{~F}$ arterial cannula (Edwards Lifesciences, Irvine, CA, USA) follows the same principle. Echo control of guide wire position in the descending aorta is also crucial. The arterial cannula is safely secured to prevent any dislocation. CPB is initiated. An additional $14 \mathrm{~F}$ Fogarty catheter (Edwards Lifescience, Irvine, CA, USA) is also inserted through the inferior vena cava (IVC) into the RA.

\section{Thoracotomy and visualization of the TV}

Being on $\mathrm{CPB}$, a limited right antero-lateral thoracotomy is performed. Lungs are deflated and the RA is identified by pushing on the atrial wall with a long forceps and echo control. Both Fogarty catheters are inflated with saline and retracted under echo control into the SVC and IVC to induce total CPB (Figure $4 A, B$ ). The pericardium is not dissected from the RA, but incised directly. Using a retractor, the TV is visualized and repair is being performed on the beating heart.

\section{TV repair}

Today our standard device for TV repair is the CosgroveEdwards annuloplasty band (Edwards Lifesciences, Irvine, CA, USA). We have demonstrated in our experience that bands are more favorable compared to rigid rings in the tricuspid position (7). Annuloplasty sutures are placed in a routine fashion, avoiding any interference with the myocardial conduction system. A $28 \mathrm{~mm}$ band was used in this case. Intra-operative direct evaluation of the repair demonstrated only mild residual regurgitation due to the right ventricular pacing wire. The RA is closed in a standard fashion.

The patient is weaned from $\mathrm{CPB}$ and the surgical result of the repair is assessed by TOE. Appropriate valve function was confirmed. The groin vessels are finally decannulated. Following protamine administration, a curved chest tube is inserted into the right pleural cavity and the thoracotomy and the groin incision are closed in layers.

The additional venous neck cannula is removed with temporary venous compression. 

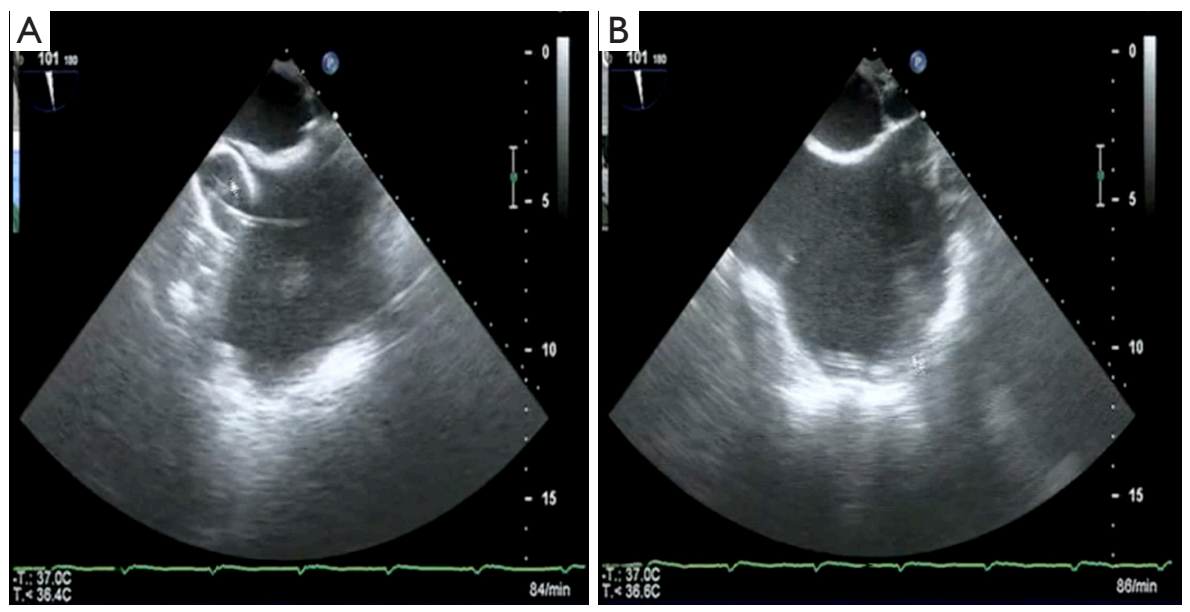

Figure 4 Intra-operative transesophageal echocardiography confirming adequate placement of Fogarty catheters in the superior (A) and inferior (B) vena cava for inducing total cardio-pulmonary bypass.

\section{Comments}

Here, we present a case of minimally invasive TV repair in a patient with previous mitral valve replacement. The minimally approach offers specific advantages in this high risk patient. Avoidance of median sternotomy with the risk of right atrial and/or ventricular damage and only limited dissection of the situs decrease the risk of bleeding, which is considerable high in patients with chronic congestive right heart failure and impairment of liver function. To further avoid dissection of the superior and inferior vena cava, two Fogarty catheters were placed in the RA via the SVC and IVC. By TOE guided inflation and retraction of the Fogarty catheters into these vessels, total CPB was initiated. With this technique, standard TV repair was performed on a beating heart.

Our own experience with this series had been published before. We have demonstrated very good perioperative outcomes in these high risk patients (5). Out of 48 patients in this series, overall 30 -day mortality was $4.2 \%$. However, in elective cases $(n=38,79 \%), 30$-day mortality was zero. This discrepancy was also true for mid-term survival. In elective cases, 5 -year survival was $72.2 \% \pm 1.0 \%$. After urgent re-operative TV surgery, however, 5-year survival was only $42.0 \% \pm 17.6 \%$.

Our favorable early results may be partially related to the minimally invasive access. Avoidance of extensive dissection not only to minimize the risk of bleeding but also of postoperative dilatation of the right side of the heart, may prevent postoperative right heart failure, which was a rare complication in our series. Using Fogarty catheters to block the SVC and IVC enables the initiation of total CPB.

It becomes clear that an extensive expertise in TOE cardiography is of immense importance to control not only the cannulation process, but also the adequate position of the Fogarty catheters in the SVC and IVC.

There are some limitations to use this technique. In cases where severe adhesions of the right pleural cavity are present, the minimally approach may not be feasible. Also in cases of unsuitable femoral arteries for arterial cannulation, alternative cannulation sides, as the axillary artery, may be used. If the venous neck cannula cannot be placed due to inadequate diameter of the internal jugular vein or an occluded vein, direct cannulation of the SVC through a slightly extended incision may be an option. The Fogarty catheter can usually still be inserted via a jugular or subclavian vein in these cases.

\section{Acknowledgements}

None.

\section{Footnote}

Conflicts of Interest: The authors have no conflicts of interest to declare.

\section{References}

1. Mohr FW, Falk V, Diegeler A, et al. Minimally invasive 
port-access mitral valve surgery. J Thorac Cardiovasc Surg 1998;115:567-74; discussion 574-6.

2. Seeburger J, Borger MA, Falk V, et al. Minimal invasive mitral valve repair for mitral regurgitation: results of 1339 consecutive patients. Eur J Cardiothorac Surg 2008;34:760-5.

3. Misfeld M, Mohr FW. Video-atlas on minimally invasive mitral valve surgery - the Mohr technique. Ann Cardiothorac Surg 2013;2:825-7.

4. Davierwala PM, Seeburger J, Pfannmueller B, et al. Minimally invasive mitral valve surgery: "The Leipzig experience". Ann Cardiothorac Surg 2013;2:744-50.

Cite this article as: Misfeld M, Davierwala P, Banusch J, Ender J, Mohr FW, Pfannmüller B. Minimally invasive, beating heart tricuspid valve surgery in a redo case. Ann Cardiothorac Surg 2017;6(3):290-293. doi: 10.21037/acs.2017.05.10
5. Pfannmüller B, Misfeld M, Borger MA, et al. Isolated reoperative minimally invasive tricuspid valve operations. Ann Thorac Surg 2012;94:2005-10.

6. Pfannmüller B, Davierwala $\mathrm{P}$, Misfeld M, et al. Postoperative outcome of isolated tricuspid valve operation using arrested-heart or beating-heart techniques. Ann Thorac Surg 2012;94:1218-22.

7. Pfannmüller B, Doenst T, Eberhardt K, et al. Increased risk of dehiscence after tricuspid valve repair with rigid annuloplasty rings. J Thorac Cardiovasc Surg 2012;143:1050-5. 